\title{
FILARIASIS DIAGNOSED BY REAL TIME SONOGRAPHIC IMAGING AS FILARIAL DANCE SIGN: A CASE REPORT
}

\author{
Shrestha $\mathcal{S}^{1}$, Shrestha $R^{2}$, Gokhale $S^{2}$
}

${ }^{1}$ Department of Radiology, Padma Nursing Home, Pokhara, Nepal, ${ }^{2}$ Department of Microbiology, MCOMS, Pokhara, Nepal.

\begin{abstract}
Lymphatic filariasis is a major public health problem. World Health Organization (WHO) has graded filariasis, as the second leading cause for permanent and long-term disability after leprosy. It is a chronic disabling parasitic disease prevalent in South Asia and Africa. A case of 26-year-old male resident of district Palpa presented with swelling of left inguinal region since 3-4 days and pain in inguinal region since a month. He had no history of trauma, fever or any other systemic symptoms. High frequency, high-resolution scrotal ultrasonography is a valuable technique for diagnosing scrotal filarial infection. This method revealed "Filarial Dance Sign" which is a distinctive and continuous pattern of movement of live microfilaria indicating active infection. It is a simple, rapid, sensitive and noninvasive technique. Noninvasive ultrasonography is preferable to the invasive FNAC.
\end{abstract}

\section{KEYWORDS}

Lymphatic filariasis, high resolution ultrasonography, filarial dance sign, noninvasive

\section{CORRESPONDING AUTHOR}

Dr. Sumiran Shrestha

Department of Radiology,

Padma Nursing Home,

Pokhara, Nepal.

Email: shresthasumiran@gmail.com 


\section{INTRODUCTION}

Lymphatic filariasis is a major public health problem in many tropical countries. World Health Organization (WHO) has graded filariasis, as the second leading cause for permanent and long-term disability after leprosy. ${ }^{1}$ It is a chronic disabling parasitic disease prevalent in South Asia and Africa. ${ }^{2}$ The disease mainly affects the lymphatics of lower limbs, spermatic cord, epididymis and retroperitoneum. ${ }^{3}$ It is diagnosed by detecting the larva (microfilaria) in blood, fluids, needle aspirates, and tissues or rarely by adult worm in the lymph node biopsy. ${ }^{4}$ Filariasis was considered as eradicable or potentially eradicable disease by International Task force for Disease Eradication. ${ }^{5}$ Here we present the case of filariasis of spermatic cord presenting as "filarial dance sign" on real time sonography.

\section{CASE PRESENTATION}

A 26 year old male resident of Palpa District presented to the Surgery OPD of Padma Nursing Home in Pokhara Municipality with the chief complaint of swelling of left inguinal region since 3-4 days and pain in inguinal region since a month. He had no history of trauma, fever or any other systemic symptoms. He had been working in army in India since 6 years and was on tour of duty in various parts of India. He had similar inguinal pain 5 years ago and responded to non-prescription analgesics.

General examination revealed an apparently healthy young man of average built. There was no anemia, jaundice, clubbing or generalized lymphadenopathy. Clinical examination revealed an elongated swelling in the left side of scrotum measuring $3.0 \times 1.0 \mathrm{~cm}$ and appreciated as beaded spermatic cord. There was mild hydrocele on the left side but no inguinal lymphadenopathy. Differential diagnosis of filariasis, inflammatory nodules, neoplasm, hydrocele and inguinal hernia were entertained. ${ }^{6}$

Hematological parameters (hemoglobin, total and differential WBC count, platelet count) and biochemical parameters were within the normal limit. No parasites were seen on peripheral blood specimen collected at $10 \mathrm{pm}$ and midnight. X ray chest PA view did not reveal any abnormality.

Real time ultra sound scanning of scrotum revealed increased echogenicity and thickness of the spermatic cord with few dilated cystic spaces showing moving internal linear echoes interpreted as dancing sign. There was minimal hydrocele on the left side (Fig. 1).

Ultrasound guided fine needle aspiration cytology from the beaded spermatic cord was undertaken and specimen examined for the microfilariae. Wet mount examination revealed twirling microfilariae, confirming the clinical diagnosis (Fig. 2). No abnormality was seen on right side of the scrotum.

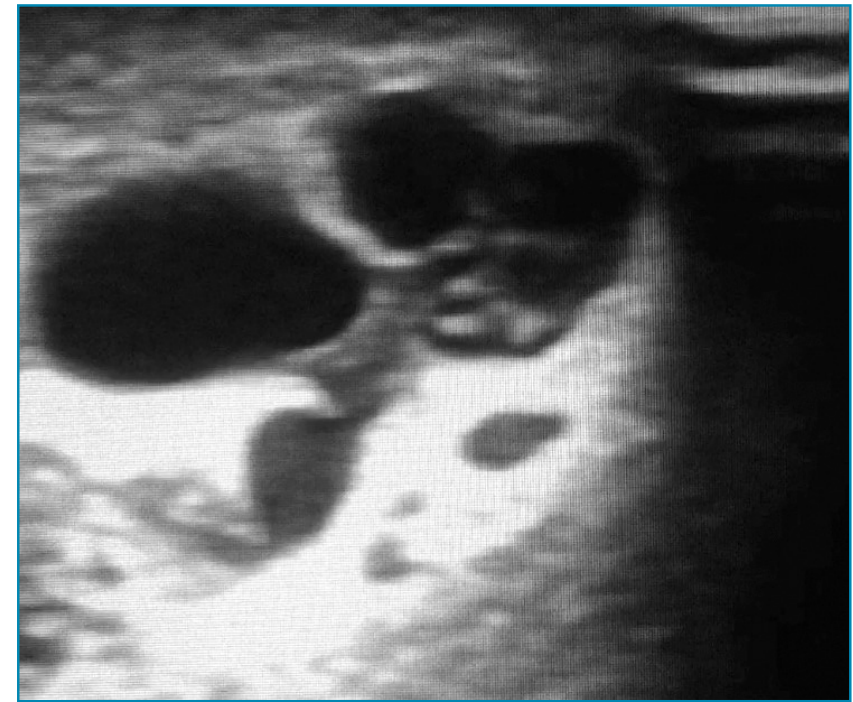

Fig. 1: Real time ultra sound scanning of scrotum revealed increased echogenicity and thickness of the spermatic cord with few dilated cystic spaces showing internal linear echoes interpreted as dancing sign.

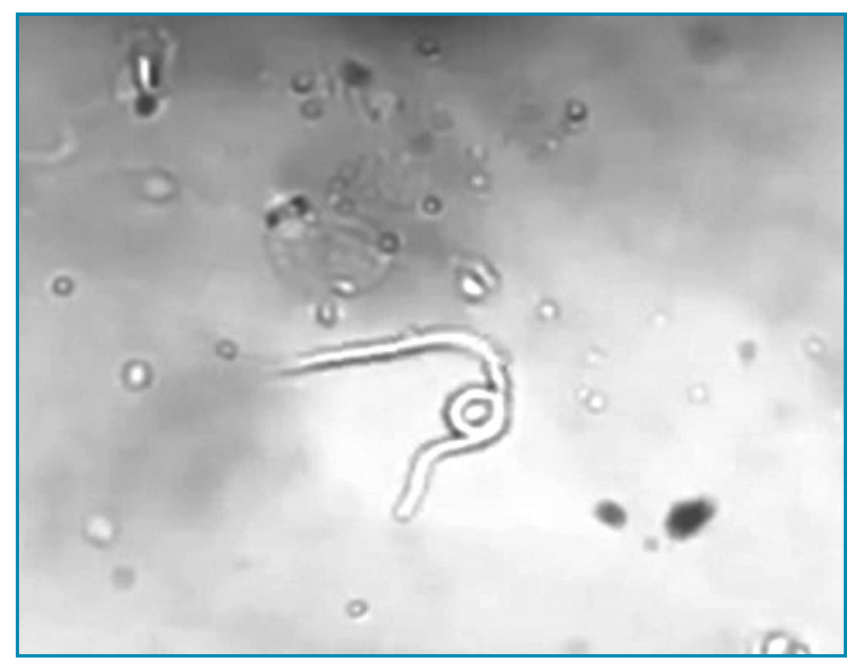

Fig. 2: Wet mount examination of fine needle aspiration cytology from the beaded spermatic cord revealed twirling microfilariae

\section{DISCUSSION}

Lymphatic filariasis (LF), caused by three species of parasitic worms (Wuchereria bancrofti, Brugia malayi and Brugia timori) is an old devastating neglected tropical diseases. ${ }^{7}$ An estimated 120 million people in 81 countries are infected currently, with 1.34 billion living in filariasis endemic areas being at risk of infection. Approximately 40 million people suffer from the stigmatizing and disabling clinical manifestations of the disease, including 15 million who have lymphoedema (elephantiasis) and 25 million men who have urogenital swelling, principally scrotal hydrocele.?

In Nepal, LF is a major public health problem in terms of morbidity, primarily due to lymphedema of arms, 
legs, breasts and genitalia, and hydrocele, hindering the socioeconomic development, social exclusion and stigmatization. ${ }^{8}$ In 2000, WHO established the Global Programme to Eliminate Lymphatic Filariasis, which has the goal of eliminating the disease as a public health problem by 2020 . $^{7}$

These methods for laboratory diagnosis of filariasis can be divided into nonspecific and specific tests. Specific tests include - direct detection of microfilaria on blood smears, urinary deposit smear, serologic tests, DNA polymerase chain reaction, ultrasonography and histopathology of the biopsy tissue. Nonspecific tests are eosinophilia, high IgE levels and lymphoscintigraphy (that reveal dilated lymph channels or backflow even in the early stage of infection). ${ }^{9}$ The latest developments in the diagnosis of lymphatic filariasis are membrane filtration method, Ultrasonography, Immunographic test ICT. ${ }^{10}$ Each of the diagnostic methods has advantages and disadvantages.

Peripheral blood smear is the gold standard for the diagnosis of filariasis. For detection of microfilariae in peripheral blood smear, the timing of blood collection should coincide with the time of peak microfilaraemia. Although this depends on the periodicity of the microfilaria in the peripheral circulation, it is most commonly in the middle of the night for nocturnally periodic LF, a time when blood sample collection is generally very inconvenient. ${ }^{11}$

Diethylcarbamazine citrate (DEC) provocation test is used in the diagnosis of filariasis. In case when sample collection is not possible to obtain night blood specimens, this method can be used. DEC flushes out the microfilariae into peripheral circulation. This test is proved to be efficient in terms of sensitivity and specificity as compared with night blood method. But due to loss of accuracy, technical and social difficulties this method is not often practiced. ${ }^{12}$ Day and night densities are highly correlated $(r=0.83)$, but the densities tend to be lower in the day blood following provocation than in the corresponding night blood, except in very light infections. ${ }^{13}$

Serological tests are the tests of choice as markers of infection, but do not confirm existing infection. These can hardly be used in individual diagnosis but is the disease is preferred option for mass screening. There might be possibility of cross-reactivity to other antigens, particularly other filarial parasites. ${ }^{14,15}$ DNA probes using real-time polymerase chain reaction (RT-PCR) are of high specificity and sensitivity, but are not cost effective. ${ }^{16}$

Membrane filtration allows for removal of elements in the blood by filtration through a membrane of desired pore size. Membrane filtration is more effectively used to determine microfilarial density than as a means of microfilaria identification. Membrane filtration method for microfilaria detection can be useful in the early stages of the disease before clinical manifestations. This method of filtering microfilariae from blood is highly sensitive, rapid and reliable, requires little equipment and is workable in the field. ${ }^{17}$ The method is cheap, quick, and simple to apply under field conditions, and it can be performed successfully without the presence of highly skilled and experienced workers. Though this can be performed quickly, it is no more sensitive than examination of the conventional blood smear.

USG guided FNAC from cystic spaces can help in collecting adequate specimen. On wet mount microscopy twirling motile microfilariae can be demonstrated. However, due to variability of ambient temperature, the motility of microfilariae may get hampered. USG guided FNAC is a highly sensitive, rapid, simple and effective diagnostic modality. ${ }^{18}$ But a disadvantage is this is the invasive way for diagnosis.

In this case, there was no evidence of lymphoedema of lower limbs (except the swelling of scrotum) or regional lymphadenopathy. Eosinophilia, a common manifestation of parasitemia was absent. Peripheral smear examination collected at expected nocturnal parasitemia did not reveal microfilaria. High index of suspicion is required to diagnose filariasis especially in the absence of eosinophilia and absence of parasite in peripheral smear. Absence of microfilariae in peripheral smear does not exclude filarial infection.

In conclusion, high frequency, high-resolution scrotal ultrasonography is a valuable technique for diagnosing scrotal filarial infection. The appearance of motile filarial worms on ultrasonography correlates with active release of microfilariae into the lymphatic vessels and hence indicates active infection. This is the only diagnostic modality that can show live adult filarial worms; therefore, it may be the preferable technique for diagnosis.

High-resolution, real time ultrasound revealed "Filarial Dance" a distinctive and continuous pattern of movement of live microfilaria. Noninvasive ultrasonography is preferable to the invasive FNAC. It is a simple, rapid, sensitive and noninvasive method of diagnosis of scrotal filarial infection. "Filarial dance sign" on ultrasonography indicates active infection. In view of ability of ultrasonography, this method should be preferred.

\section{REFERENCES}

1. Dayal A, Selvaraju K. Filariasis of The Breast: Webmed Central Surgery 2010; 1: WMC00942.

2. Sinha R, Sengupta S, Pal S, Adhikari A. Incidental diagnosis of filariasis in association with carcinoma of gall bladder: Report of a case evidenced on ultrasound-guided fine-needle aspiration cytology with review of the literature. J Cytol 2014; 31:174-5. doi: 10.4103/0970-9371.145662.

3. Kaur R, Phillip KJ, Masih K. Johnny C. Filariasis of the Breast Mimicking Inflammatory Carcinoma. Lab Med 2009; 40: 683-5. 
4. Myageri A. Wuchereria Bancrofti adult worms in fine needle aspirate: A case report. J Cytol 2006; 23: 91-3.

5. Sabesan S, Raju HK, Srividya A, Das PK. Delimitation of lymphatic filariasis transmission risk areas: A geo-environmental approach. Filaria J 2006; 5: 12.

6. Micheal M. A case report inflammatory nodule of right spermatic cord due to filarial broncrofti. Can Med Assoc J 1968; 99: 765-6.

7. WHO Global Programme to Eliminate Lymphatic Filariasis (GPELF). Progress Report 2000-2009 and Strategic Plan 2010- 2020. Dec. 2010 Geneva.

8. DoHS (2013) Annual Report 2068/2069 (2011/2012). Kathmandu: Department of Health Services, Ministry of Health and Population, Government of Nepal.

9. Ghosh US. Filariasis: Making an Early diagnosis. Med Update 2012; 22: 20-3.

10. Nateshan A, Venkateswara RT. Lymphatic Filariasis: Drug targets and Nematicidal Plants. J Pharm Sci Res 2015; 7: 928-33.

11. McMahon JE. The examination--time/dose interval in the provocation of nocturnally periodic microfilariae of Wuchereria bancrofti with diethylcarbamazine and the practical uses of the test. Tropenmed Parasitol 1982; 33: 28-30.

12. Wijeyaratne PM, Singha P, Verma OP, Motha B. Evaluation of the diethylcarbamazine provocative test in the diagnosis of Wuchereria bancrofti infections in the Nigerian savanna and the effects on Dipetalonema perstans. Trans Roy Soc Trop Med Hyg 1982; 76: 387-91.

13. McMahon JE, Marshall TF, Vaughan, JP, Kolstrup, N. Tanzania filariasis project: a provocative day test with diethylcarbamazine for the detection of microfilariae of nocturnally periodic Wuchereria bancrofti in the blood. Bull. World Health Organ 1979; 57: 759-65.

14. Chanteau S, Glaziou P, Moulia-Pelat JP, Plichart C, Luquiaud P, Cartel JL. Low Positive Predictive Value of anti-Brugia Malayi IgG and IgG4 Serology for the Diagnosis of Wuchereria Bancrofti. Trans Roy Soc Trop Med Hyg 1994; 88: 661-2.

15. Harnett W, Bradley JE, Garate T. Molecular and Immunodiagnosis of Human Filarial Nematode Infections. Parasitology 1998; 117 Suppl: S59-S71.

16. Nuchprayoon S. DNA-based Diagnosis of Lymphatic Filariasis. Southeast Asian J Trop Med Public Health. 2009; 40: 904-13.

17. Denham DA, Dennis DT. Ponnudurai, T, Nelson, GS. Guy F. Comparison of a counting chamber and thick smear methods of counting microfilariae. Trans Roy Soc Trop Med Hyg 1971; 65: 521-6.

18. Dey S, Datta S, Snehamay C, Prabir PC, Binny K, Mandal S. Preoperative Ultrasound Guided Fine Needle Aspiration Cytology of Ovarian Lesions - Is It a Rapid and Effective Diagnostic Modality? J Clin Diagn Res 2016; 10: EC16-EC19. 